\title{
REDUCED COMPLEXITY MIMO CHANNEL ESTIMATION AND EQUALIZATION USING A POLYNOMIAL-PREDICTOR-BASED VECTOR GLMS ALGORITHM
}

\author{
Yau Hee Kho and Desmond P. Taylor \\ Department of Electrical and Computer Engineering, \\ University of Canterbury, \\ Christchurch, New Zealand. \\ Email: $\{$ yhkho, taylor\}@elec.canterbury.ac.nz
}

\begin{abstract}
A reduced complexity multiple-input multiple-output (MIMO) channel estimator known as the polynomial-predictor-based vector generalized least mean squares (VGLMS) estimator is developed. It is a simplification of a previously developed polynomial-predictor-based vector generalized recursive least squares (VGRLS) estimator, achieved by replacing the online recursive computation of the 'intermediate' matrix by an offline pre-computed matrix. Similar to the VGRLS estimator, it is able to operate in Rayleigh or Rician fading environments without reconfiguration of the state transition matrix to accommodate the non-random mean components. It is seen to offer a trade-off between reduced complexity channel estimation and good system performance.
\end{abstract}

\section{INTRODUCTION}

A multiple-input multiple-output (MIMO) channel estimator employing a polynomial-predictor-based vector generalized recursive least squares (VGRLS) algorithm was developed in [1]. The VGRLS estimator is capable of tracking time-varying frequency selective channel responses in a Rayleigh or Rician fading environment, and its performance can be made to approach that of an optimum estimator employing a Kalman filter [1]. It is a vector extension of the polynomial-based generalized recursive least squares (GRLS) algorithm of [2]. A simplification of the GRLS algorithm was developed in [3] where the online recursive computation of the 'intermediate' matrix is replaced by an offline pre-computed matrix. The resulting algorithm is known as the polynomial-based generalized least mean squares (GLMS) algorithm.

In this paper we investigate simplification of the VGRLS algorithm following a similar approach to [3]. The resulting algorithm is a polynomial-predictor-based vector generalized least mean squares (VGLMS) algorithm. We evaluate its performance in terms of the 'mean square deviation' (MSD) and symbol error rate (SER) when operating with a MIMO minimum-mean-square-error (MMSE) decision feedback equalizer (DFE) [4]. We demonstrate by simulation that the VGLMS estimator is able to operate in both Rayleigh and Rician fading environments. We also compare the relative complexity of the VGRLS and VGLMS algorithms.

In the following, we describe a general signal model in section II. In section III, we summarize the VGRLS estimator and then develop the VGLMS estimator. In section IV we present a complexity comparison and show the computational savings of VGLMS estimator. Simulation results and discussions are presented in section $\mathrm{V}$, followed by conclusions in section VI.

\section{Signal AND Channel Models}

\section{A. The General Model}

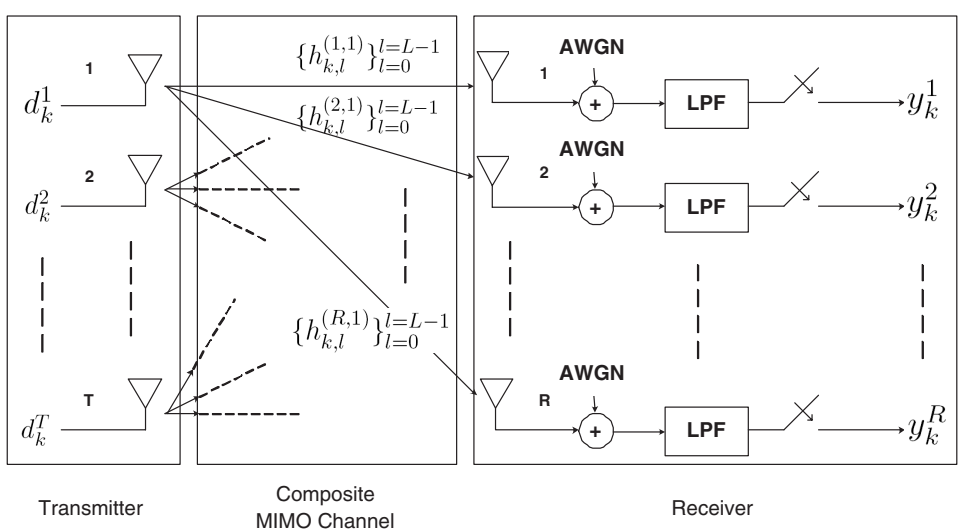

Figure 1: A general block diagram of a discrete-time MIMO communication system at time $k$ for $i=1,2, \cdots, T$ transmit and $j=1,2, \cdots, R$ receive antennas.

We assume a discrete-time model for a $(T, R)$ MIMO system with $T$ transmit and $R$ receive antennas, as shown in Fig. 1. Linearly independent signal streams are transmitted from each of the $T$ antennas in a V-BLAST [5] fashion. At the receiver, each of the $R$ antennas receives a linear combination of the transmitted signal streams. The $j$-th symbol-rate sampled complex baseband received signal at time $k$ may be written as

$$
y_{k}^{(j)}=\sum_{i=1}^{T} \sum_{l=0}^{L-1} d_{k-l}^{(i)} h_{k, l}^{(j, i)}+n_{k}^{(j)} ; j=1,2, \cdots, R
$$

where $d_{k}^{(i)}$ is the $k$-th transmitted complex baseband $M$-ary data symbol from the $i$-th antenna, $\left\{h_{k, l}^{(j, i)}\right\}_{l=0}^{l=L-1}$ represents the fading dispersive composite channel impulse response between the $i$-th transmit and $j$-th receive antenna at time $k$. We assume a delay spread of $L$ symbol periods, and note that $n_{k}^{(j)}$ is additive white Gaussian noise (AWGN) with variance, $\sigma_{n}^{2}$.

The MIMO received signal of (1) may be expressed in matrix-vector form [1] as

$$
\mathbf{y}_{k}=\mathfrak{d}_{k} \mathfrak{h}_{k}+\mathbf{n}_{k}
$$


by defining the vectors

$$
\begin{gathered}
\mathbf{y}_{k}=\left[y_{k}^{(1)}, y_{k}^{(2)}, \cdots, y_{k}^{(R)}\right]^{t} \\
\mathfrak{h}_{k}=\left[h_{k, 0}^{(1,1)} \cdots h_{k, 0}^{(R, 1)} \cdots h_{k, 0}^{(1, T)} \cdots h_{k, 0}^{(R, T)}, \cdots\right. \\
\left.h_{k, L-1}^{(1,1)} \cdots h_{k, L-1}^{(R, 1)}, \cdots, h_{k, L-1}^{(1, T)}, \cdots, h_{k, L-1}^{(R, T)}\right]^{t} \\
\mathfrak{d}_{k}=\left[d_{k}^{(1)} \cdots d_{k}^{(T)}, d_{k-1}^{(1)} \cdots d_{k-1}^{(T)}, \cdots,\right. \\
\left.d_{k-L+1}^{(1)} \cdots d_{k-L+1}^{(T)}\right] \otimes \mathbf{I}_{R} \\
\mathbf{n}_{k}=\left[n_{k}^{(1)}, n_{k}^{(2)}, \cdots, n_{k}^{(R)}\right]^{t}
\end{gathered}
$$

where $t$ denotes transposition, $\mathbf{I}_{R}$ is the $(R \times R)$ identity matrix and $\otimes$ is the Kronecker product.

\section{B. Channel Model}

The RT MIMO sub-channels are assumed to exhibit wide sense stationary uncorrelated scattering (WSSUS) [6]. We assume that each of the RTL channel coefficient, $h_{k, l}^{(j, i)}$, evolves according to Clarke's fading model [7] under the same fading conditions. We also assume that each consists of a non-random (specular) component and a random (diffuse) component such that $h_{k, l}^{(j, i)}=h_{l}^{(n r),(j, i)}+h_{k, l}^{(r),(j, i)}$. The power ratio between the specular and the diffuse components is given by the Rice $K$-factor,

$$
K=\frac{\left|h^{(n r)}\right|^{2}}{E\left\{\left|h^{(r)}\right|^{2}\right\}}
$$

where a $K$ value of 0 corresponds to Rayleigh fading and a large $K$ corresponds to Rician fading. In reality a specular component can be present in all or any of the delay paths and the value of the $K$-factor can be the same or different for each path. For simplicity, we assume that all multipath components contain specular components with the same value of $K$.

\section{ChanNEL Estimation}

\section{A. The VGRLS estimator}

In [1], a state-space model of the channel state vector with unforced dynamics based on polynomial predictors of length, $P$, and order, $N$, was developed as

where

$$
\mathbf{h}_{k+1}=\mathbf{U h}_{k}
$$

$$
\mathbf{h}_{k}=\left[\mathfrak{h}_{k}^{t}, \mathfrak{h}_{k-1}^{t}, \cdots, \mathfrak{h}_{k-P+1}^{t}\right]^{t}
$$

is the $(R T L P \times 1)$ channel state vector at time $k$ and the (RTLP $\mathrm{x} R T L P$ ) state transition matrix is given by

$$
\mathbf{U}=\left(\begin{array}{ccccc}
\mathfrak{U}_{1} & \mathfrak{U}_{2} & \cdots & \mathfrak{U}_{P-1} & \mathfrak{U}_{P} \\
& \mathbf{I}_{R T L(P-1)} & & & \mathbf{0}_{R T L(P-1), R T L}
\end{array}\right)
$$

Table 1: Polynomial Coefficients of Various Order and Length

\begin{tabular}{|c|c|c|}
\hline Length P & Order $\boldsymbol{N}$ & Polynomial Coefficients $\left\{a_{1}, a_{2}, \cdots, a_{P}\right\}$ \\
\hline \hline 2 & 0 & $\{1 / 2,1 / 2\}$ \\
2 & 1 & $\{2,-1\}$ \\
3 & 0 & $\{1 / 3,1 / 3,1 / 3\}$ \\
3 & 1 & $\{4 / 3,1 / 3,-2 / 3\}$ \\
3 & 2 & $\{3,-3,1\}$ \\
\hline
\end{tabular}

where $\mathbf{0}_{m, n}$ is the ( $\left.m \times n\right)$ null matrix. Each $(R T L \times R T L)$ polynomial predictor matrix is given by $\mathfrak{U}_{r}=a_{r} \mathbf{I}_{R T L}$, where $a_{r}$ is the $r$-th tap weight of a polynomial predictor of length $P$ and order $N$. These are independent of channel statistics and the coefficients for various orders and lengths are shown in Table 1 as calculated in [2].

The VGRLS algorithm is then given by [1]

$$
\begin{gathered}
\hat{\mathbf{h}}_{k / k-1}=\mathbf{U} \hat{\mathbf{h}}_{k-1 / k-1} \\
\mathbf{P}_{k / k-1}=\lambda^{-1} \mathbf{U} \mathbf{P}_{k-1 / k-1} \mathbf{U}^{H} \\
\mathbf{K}_{k}=\mathbf{P}_{k / k-1} \mathbf{d}_{k}^{H}\left(\mathbf{I}_{R}+\mathbf{d}_{k} \mathbf{P}_{k / k-1} \mathbf{d}_{k}^{H}\right)^{-1} \\
\mathbf{P}_{k / k}=\left(\mathbf{I}_{R T L P}-\mathbf{K}_{k} \mathbf{d}_{k}\right) \mathbf{P}_{k / k-1} \\
\hat{\mathbf{h}}_{k / k}=\hat{\mathbf{h}}_{k / k-1}+\mathbf{K}_{k}\left(\mathbf{y}_{k}-\mathbf{d}_{k} \hat{\mathbf{h}}_{k / k-1}\right) .
\end{gathered}
$$

with $\mathbf{d}_{k}=\left[\begin{array}{c|c}\mathfrak{d}_{k} & \mathbf{0}_{R, R T L(P-1)}\end{array}\right]$.

To initialize the algorithm and to ensure that $\mathbf{P}_{k / k}^{-1}$ is nonsingular for all $k$, we set $\mathbf{P}_{1 / 0}=\delta^{-1} \mathbf{I}_{R T L P}$ where $\delta$ is a small real positive constant and $\hat{\mathbf{h}}_{1 / 0}=0_{R T L P, 1}$.

\section{B. The reduced complexity estimator}

There are two recursions involved in the VGRLS algorithm. One predicts the channel responses recursively as in (11) and (15); the other computes the 'intermediate' matrix ${ }^{1}, \mathbf{P}_{k / k}$, in (12) and (14). In the reduced complexity case of this paper, it is approximated [3] by a static replacement, $\hat{\mathbf{P}}=$ $\left(\lim _{k \longrightarrow \infty} E\left[\mathbf{P}_{k / k}^{-1}\right]\right)^{-1}$. This is the inverse of the steady-state mean of the correlation matrix [3]. To evaluate $\hat{\mathbf{P}}$ requires only the knowledge of the autocorrelation matrix of the data vector $\mathbf{R}_{\mathbf{d}}=E\left[\mathbf{d}_{k}^{H} \mathbf{d}_{k}\right]$, the state transition matrix $\mathbf{U}$ and the "forget factor" $\lambda$. These parameters may usually be specified $a$ priori [8]. In general a closed form expression for $\hat{\mathbf{P}}$ cannot be obtained. Instead, we compute $\hat{\mathbf{P}}_{k / k}$ using the offline recursion [3]

$$
\begin{gathered}
\hat{\mathbf{P}}_{k / k-1}=\lambda^{-1} \mathbf{U} \hat{\mathbf{P}}_{k-1 / k-1} \mathbf{U}^{H} \\
\Psi_{k / k-1}=\left(\mathbf{I}_{R T L P}+\mathfrak{D} \hat{\mathbf{P}}_{k / k-1} \mathfrak{D}^{H}\right)^{-1}
\end{gathered}
$$

${ }^{1} \mathbf{P}_{k / k}$ is the inverse input autocorrelation matrix in a conventional RLS algorithm. 
Table 2: Number of real operations per iteration of the VGRLS and VGLMS algorithms for a $(2,2)$ MIMO system with a delay spread, $L=3$ and a predictor length, $P=3$.

\begin{tabular}{|c|c|c|c|}
\hline Algorithm Type & $\boldsymbol{x}$ & + & / \\
\hline \hline VGRLS & 400608 & 387288 & 4 \\
VGLMS & 14400 & 11448 & 4 \\
\hline
\end{tabular}

$$
\hat{\mathbf{P}}_{k / k}=\hat{\mathbf{P}}_{k / k-1}-\hat{\mathbf{P}}_{k / k-1} \mathfrak{D}^{H} \Psi_{k / k-1} \mathfrak{D} \hat{\mathbf{P}}_{k / k-1}
$$

where $\mathfrak{D}$ is a $(R T L P \times R T L P)$ matrix, such that $\mathbf{R}_{\mathrm{d}}=\mathfrak{D D}^{H}$, and $\hat{\mathbf{P}}_{0 /-1}=\mathbf{P}_{0 /-1}$. The steady-state approximation of the inverse correlation matrix is then $\hat{\mathbf{P}}=\hat{\mathbf{P}}_{k / k}$ for large values of $k$. For the special case of $N=0$ and $P=1$, the static approximation has the closed form $\hat{\mathbf{P}}=(1-\lambda) \mathbf{R}_{\mathbf{d}}^{-1}$.

Recognizing that the Kalman gain becomes $\mathbf{K}_{k}=\mathbf{P}_{k / k} \mathbf{d}_{k}^{H}$ [9] and replacing $\mathbf{P}_{k / k}$ by $\hat{\mathbf{P}}$ in (11) - (15), we obtain the $k$-th recursive update equation that describes the reduced complexity estimation algorithm as

$$
\hat{\mathbf{h}}_{k+1 / k}=\mathbf{U}\left[\hat{\mathbf{h}}_{k / k-1}+\hat{\mathbf{P}} \mathbf{d}_{k}^{H}\left(\mathbf{y}_{k}-\hat{\mathbf{y}}_{k / k-1}\right)\right] .
$$

This is readily implemented. We note that in the special case where $N=0, P=1$ and $\mathbf{R}_{\mathbf{d}}=\mathbf{I}_{R T L P}$, (19) reduces to a standard vector LMS algorithm with LMS step size, $\mu=1-$ $\lambda$. The algorithm of (19) is the desired polynomial-predictorbased vector generalized least mean square (VGLMS) channel estimator following [3].

\section{A COMPLEXITY COMPARISON OF THE VGRLS AND VGLMS ALGORITHMS}

We compare the complexity of the VGRLS and VGLMS algorithms for the following scenario: a $(2,2)$ MIMO system with $L=3$ multipath rays in each of the sub-channels and a predictor length of $P=3$. Table 2 gives the number of real operations $^{2}$ needed per iteration of the algorithms. It is clear from Table 2 that substantial computational savings can be achieved by the VGLMS algorithm. This is because the online recursion of (14) for computing $\mathbf{P}_{k / k}$ requires $\mathcal{O}\left((R T L P)^{3}\right)$ in the highest term of calculations. In fact this constitutes the bulk of the computational complexity and by getting rid of this online recursion, we reduce the complexity of the algorithm to just $\mathcal{O}\left((R T L P)^{2}\right)$. The savings become more significant as the dimension of the MIMO system increases.

\section{Simulations Results and Discussion}

We now consider the performance of the VGLMS channel estimator. We follow the approach of [1] and assume an uncoded, VBLAST-type [5], MIMO system operating in a spatial multiplexing mode. We assume independent sub-channels each modelled by Clarke's fading model [7] and simulated as

\footnotetext{
${ }^{2}$ We assume that one complex multiplication requires 4 real multiplications and 2 real additions; while one complex addition requires 2 real additions.
}

in [10]. Each sub-channel is assumed to consist of $L=3$ multipath rays and to have a uniform power-delay profile with the delay between adjacent rays symbol-spaced so that total maximum delay spread $\tau_{\max }=3 T$. This represents a normalized delay spread, $\tau_{\max } / T$, of 3 and a reasonably dispersive fading environment. Each of the multipath rays contains a specular and a random component. The Rice $K$-factor of (7) defines the power ratio between the specular and random components.

QPSK signal streams are transmitted from each transmit antenna. A raised cosine filter with $50 \%$ roll-off factor is used at the transmitter with its response truncated to $\pm 2 T$. An ideal low pass filter with sufficient bandwidth to accommodate the Doppler faded signal is employed at the receiver. Each frame at each antenna consists of $L_{t}=26$ training symbols and $L_{d}=$ 116 data symbols, unless stated otherwise.

We evaluate the performance of the VGLMS estimator in terms of the 'mean square deviation' (MSD) which is the average squared norm difference between the original and the estimated channel responses. The estimator is operated alone and constantly updates the estimated channel responses using knowledge of the transmitted signals. It is assumed to operate in transient mode during training, after which it operates in steady state mode. The MSD measures the performance of the estimator in steady state and for this purpose, the first $L_{t}$ symbols of each frame are not included in the evaluation. At the beginning of a frame the estimator re-initializes, and starts acquisition again. The MSD following the $\alpha$-th acquisition is defined as

$$
\sigma_{M S D}^{2}(\alpha)=\left\langle\left\|\mathfrak{h}_{k}-\hat{\mathfrak{h}}_{k / k-1}\right\|^{2}\right\rangle
$$

where $\langle\| .||\rangle$ denotes the average of the euclidean norm operator. It is accumulated and averaged for 10,000 frames for each SNR point and the overall MSD is then averaged across all $R T$ sub-channels.

The SNR is defined per received antenna. Given that $\sigma_{n}^{2}$ is the AWGN variance at the input of each receiver, with the QPSK signals and the overall random components of the multipath rays normalized to unit energy, we have

$$
S N R=10 \log \left(\frac{(1+K)}{\sigma_{n}^{2}}\right) .
$$

Unless stated otherwise, we assume that the total transmitted power is restricted to unit power and allocated equally between the $T$ antennas.

Fig. 2 shows the MSD behavior of the VGLMS estimator for a $(2,2)$ MIMO system in a Rayleigh fading environment with a normalized fade rate of $f_{D} T=0.002$ where $f_{D}$ is the maximum Doppler frequency and $\lambda=0.95$. The performance of the VGLMS estimator with a polynomial predictor of order $N=0$ is similar to that of a conventional vector LMS estimator, where the MSD remains high regardless of the SNR. This shows that a conventional LMS estimator is not able to track fast fading very well. A polynomial order of $N=1$ offers slightly lower MSD. However, the MSD remains high regardless of the length of the training sequence used, even for $L_{t}=200$ at high SNR. Significantly lower MSD is achieved with an estimator using 


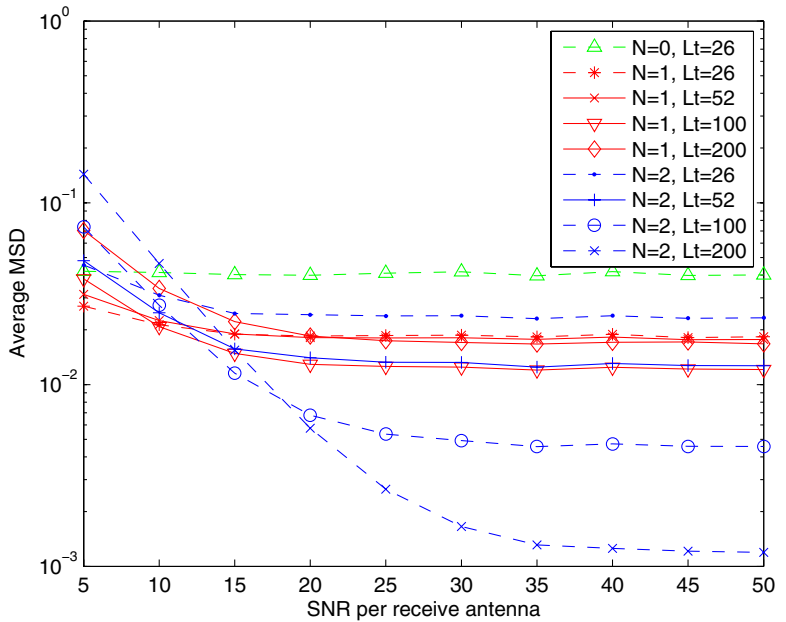

Figure 2: MSD behavior of the VGLMS estimator for a $(2,2)$ MIMO system for Rayleigh fading with a normalized fade rate $f_{D} T=0.002$ and $\lambda=0.95$. VGLMS estimator with a polynomial order of $N=0$ corresponds to a conventional vector LMS algorithm. With sufficient training sequence length, the MSD of VGLMS estimator with a polynomial order of $N=2$ can be made sufficiently low.

a polynomial order of $N=2$ and a training sequence length of just $L_{t}=100$. When the training sequence length is increased to $L_{t}=200$, the MSD can be made to approach $10^{-3}$ at high SNR. However, this MSD performance is relatively poor when compared to that of a VGRLS estimator which gives an MSD of less than $10^{-4}$ at high SNR with $L_{t}=78$. Although not shown here, we have found that MSD behavior is in general much better at a lower fade rate. For example at a normalized fade rate of 0.0001 , the MSD for a polynomial order of $N=1$ with $L_{t}=200$ approaches $10^{-3}$ while that for $N=2$ with $L_{t}=$ 200 approaches $10^{-4}$ at high SNR.

We have also evaluated the error rate performance of a receiver consisting of a VGLMS estimator and a vector DFE [4] operating in a decision-directed mode using the immediate previously detected symbols as feedback. This means the estimated channel responses from the VGLMS estimator are used to calculate the tap coefficients of the DFE and the outputs from the DFE are used by the VGLMS estimator to update the estimated channel responses. The DFE consists of 4 forward and 2 feedback matrix-taps with a decision delay of 3 symbols. Each frame consists of $L_{t}=200$ training and $L_{d}=1160$ data symbols. The simulation at each SNR point is carried out until 200 symbol errors are encountered, and the symbol error rate (SER) is averaged across the $T$ transmitted signal streams.

The average SER performance of a $(2,2)$ system in a Rayleigh fading with a normalized fade rate of 0.002 is shown in Fig. 3. We include the performance of a vector DFE using a Kalman estimator, and a vector DFE having perfect channel information for reference. In general the system performance using the VGLMS estimator is worse than that when using the optimum Kalman estimator. However, it is much better than that using a conventional vector LMS estimator (curve for $N=$

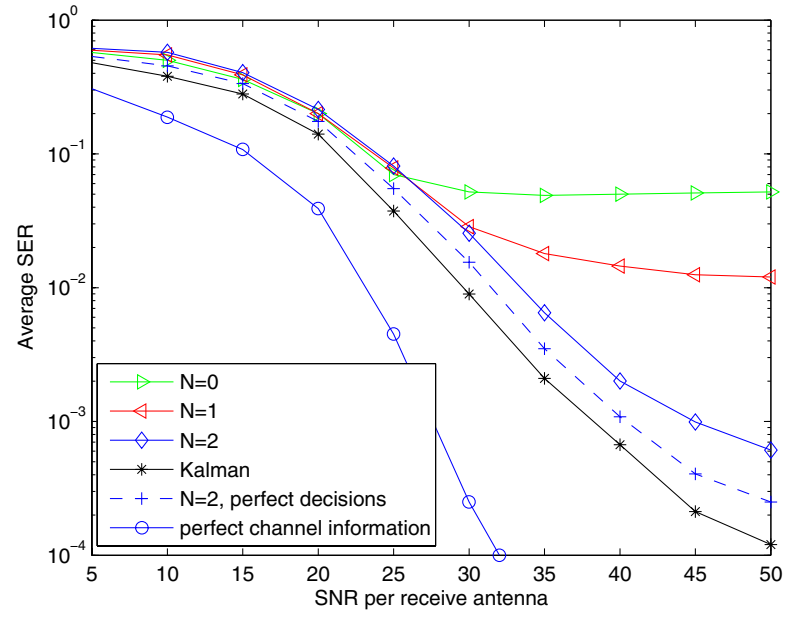

Figure 3: Average SER performance of a $(2,2)$ MIMO system using a VGLMS estimator and a Kalman estimator, each operating with a vector DFE in a Rayleigh fading with a normalized fade rate of 0.002 . The VGLMS estimator has a predictor length of $P=3$ and various orders as shown. With perfect decisions for $N=2$, the transmitted signals instead of the outputs from the DFE, are used by the VGLMS estimator.

$0)$. For $N=2$, the estimator's performance is about $7 \mathrm{~dB}$ away from that of the Kalman estimator at an SER of $10^{-3}$. When perfect decisions instead of the output decisions from the DFE are used by the estimator, the difference is reduced to about 2 $3 \mathrm{~dB}$ at the same SER.

For comparison, we have plotted in Fig. 4 the error performance curves of the VGLMS, VGRLS [1] and Kalman estimator [1] operating with the above vector DFE in a $(2,2)$ MIMO system in Rayleigh fading with normalized fade rates of 0.002 and 0.0001 . At a fade rate of 0.002 , the VGLMS based system is degraded between 6 and $7 \mathrm{~dB}$ at an SER of $10^{-3}$ compared to systems using the VGRLS and Kalman estimators respectively. The degradation is due to the simplification in the estimation process. However, in slower fading, i.e. at a normalized fade rate of 0.0001 , the VGLMS estimator performs much better since the channel is easier to track. The SER at $10^{-3}$ is improved by about $5 \mathrm{~dB}$ compared to that at a fade rate of 0.002 .

Fig. 5 shows the average SER of the above receivers in a Rician fading environment. In order to compare our results with [11] we follow their approach. We allocate unit transmit power to each of the transmitters, so the resulting graph has a $\log _{10}(T)=3 \mathrm{~dB}$ increase in the SNR per antenna. A Rician $K$ factor of 10 and a normalized fade rate of 0.007 is used. We also assume the specular components of the fading channel responses to be known when simulating the Kalman filter. This simplifies the simulation by not requiring the state transition matrix to be restructured [12]. However, we have used 3 instead of 2 multipath rays in each sub-channel and this affects the vector DFE's design. We note that the resulting Kalman filter's curve is reasonably close to that of [11].

We note that at an SER of $10^{-3}$ the system using a VGLMS estimator with $N=2$ is $2-3 \mathrm{~dB}$ worse than the Kalman 


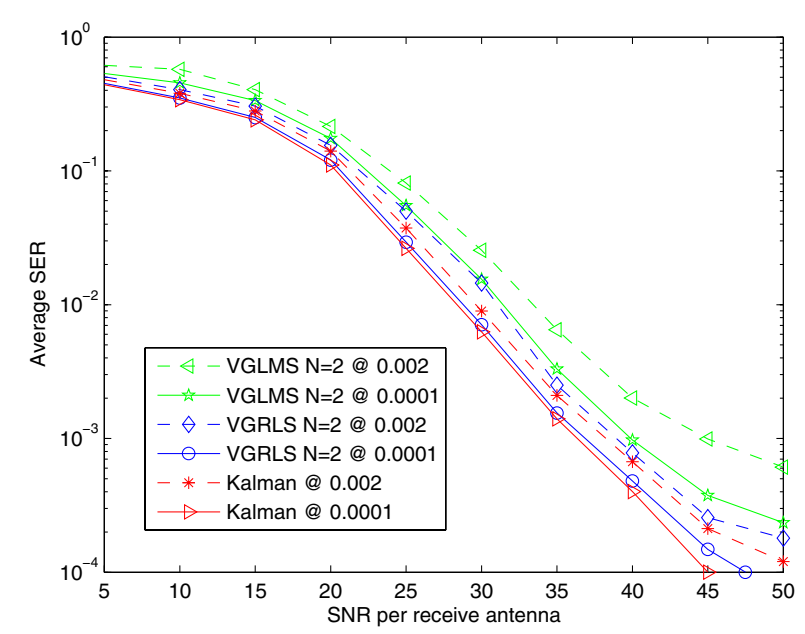

Figure 4: Average SER performance of a $(2,2)$ MIMO system using VGLMS, VGRLS and Kalman estimators operating with a vector DFE in a Rayleigh fading with normalized fade rates of 0.002 (dashed lines) and 0.0001 (solid lines) as indicated. The VGLMS and VGRLS estimators each has a predictor length of $P=3$.

estimator-based system. This margin is worse when compared to the $1 \mathrm{~dB}$ difference between a VGRLS estimator and a Kalman estimator [1]. However, the degradation is compensated by the reduced complexity in the channel estimation process. The results with perfect decision feedback to the estimator and perfect channel information are also included. They indicate almost a $10 \mathrm{~dB}$ loss with respect to perfect channel information at a SER of $10^{-3}$, but modest losses with respect to a Kalman estimator.

\section{CONCLUSIONS}

We have extended the reduced complexity GLMS estimator of [3] to a MIMO channel estimator that is capable of tracking the time-varying frequency selective channel responses in both Rayleigh and Rician fading environments. The resulting VGLMS estimator is a simplification of the previously developed VGRLS estimator [1]. It does not require channel and noise statistics to operate and does not require specific modelling of the specular component to operate in a Rician fading channel. Its performance is degraded when compared to the VGRLS estimator, however, it offers significant savings in computational load per iteration of the algorithm, especially when the MIMO dimension is large. We demonstrate that the VGLMS estimator is able to offer sufficiently good performance in slow Rayleigh fading or Rician fading with a strong mean component, where the performance is only 2 to $4 \mathrm{~dB}$ worse than that of a Kalman-estimator-based system.

\section{REFERENCES}

[1] Y. H. Kho and D. P. Taylor, "Mimo channel estimation and tracking based on polynomial prediction with application to equalization," $s u b$ mitted to IEEE Trans. Veh. Technol., 8 Feb. 2007.

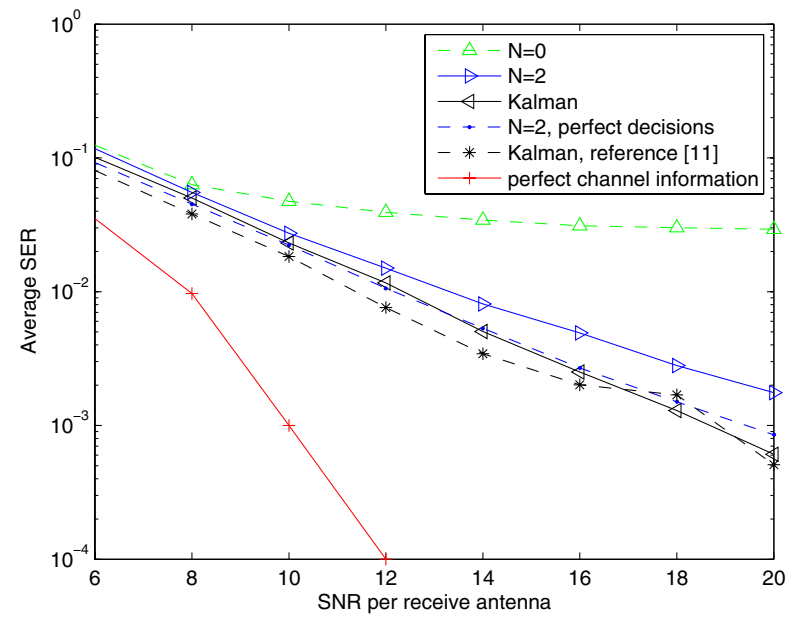

Figure 5: A $(2,2)$ MIMO average SER performance of VGLMS and Kalman estimators operating with a vector DFE in a Rician fading with a normalized fade rate of 0.007 and a $K$-factor of 10. The VGLMS estimator has a predictor length of $P=3$. The results of [11] is also plotted for reference.

[2] W. S. Leon and D. P. Taylor, "The polynomial-based generalized recursive least squares estimator for rayleigh fading channels," Proc. of GLOBECOM, vol. 5, pp. 2401-2405, 2003.

[3] _ _ "The polynomial-based generalized least mean squares estimator for rician and rayleigh fading channels," IEEE 60th VTC, vol. 6, pp. 3861-3865, 2004.

[4] N. Al-Dhahir and A. H. Sayed, "The finite-length multi-input multioutput mmse-dfe," IEEE Trans. Signal Proccessing, vol. 48, no. 10, pp. 2921-2936, Oct. 2000.

[5] G. J. Foschini, G. D. Golden, R. A. Valenzuela, and P. W. Wolniansky, "Simplified processing for high spectral efficiency wireless communication employing multi-element arrays," IEEE J. Select. Areas Commun., vol. 17, no. 11, pp. 1841-1852, Nov. 1999.

[6] P. Bello, "Characterization of randomly time-variant linear channels," IEEE Trans. Commun. Syst., vol. CS-11, no. 4, pp. 360-393, Dec. 1963.

[7] W. C. Jakes, Microwave mobile communications : Edited by William C. Jakes. N.Y.: Wiley, 1974.

[8] W. S. Leon, Equalisation and Estimation for Fading Channels. $\mathrm{PhD}$ Thesis, University of Canterbury, 2003.

[9] A. P. Clark, Adaptive Detectors for Digital Modems. London, U.K.: Pentech Press, 1989.

[10] D. Verdin and T. C. Tozer, "Generating a fading process for the simulation of land-mobile radio communications," Electronics Letters, vol. 29, no. 23, pp. 2011-2012, Nov. 1993.

[11] C. Komninakis, C. Fragouli, A. H. Sayed, and R. D. Wesel, "Multi-input multi-output fading channel tracking and equalisation using kalman estimation," IEEE Trans. Signal Proccessing, vol. 50, no. 5, pp. 1065-1076, May 2002.

[12] L. M. Davis, I. B. Collings, and R. J. Evans, "Coupled estimators for equalization of fast-fading mobile channels," IEEE Trans. Commun., vol. 46, no. 10 , pp. 1262-1265, Oct. 1998. 